\title{
Contact-Induced Nonlinearity in Oscillating Belts and Webs
}

\author{
P. F. TURNBULL \\ N. C. PERKINS \\ W. W. SCHULTZ \\ Mechanical Engineering and Applied Mechanics, University of Michigan, Ann Arbor, \\ MI 48109-2125, U.S.A.
}

(Received 13 February 1995, accepted 18 May 1995)

\begin{abstract}
This study is motivated by issues in belt dynamics and paper forming where the oscillatory motion of the contact point between the belt and pulley or the web and roll is important. The objective is to evaluate the influence of the nonlinear contact boundary conditions on the dynamics of the belt or web. To accomplish this objective, two models are analyzed using perturbation methods. The first model represents a string on an elastic foundation (an approximation of the paper forming process) and the second model represents a tensioned beam (accessory drive belt). The first correction to the fundamental natural frequency and the dynamic beluweb length are determined as a function of the governing parameters. For both models, the change in belt length is found to be proportional to the radius of the pulley/roll and the initial belv/web energy.
\end{abstract}

Key Words: Nonlinear oscillations, belts and webs, contact

\section{INTRODUCTION}

The vibration of belts and webs are often associated with a broad class of dynamical systems referred to as axially moving materials (Mote, 1972). Examples of axially moving materials include band saws, chains, belts, paper, webs, cables, vehicle tracks, and tapes. These axially moving materials are principally driven through contact with rotating pulleys, sprockets, rolls, drums, spools, and so on. Recent developments concerning the vibration and stability of axially moving material systems are reviewed in Wickert and Mote (1988).

This investigation examines the dynamic contact of a belt or web on a curved surface (e.g., pulley or roll). In particular, attention focuses on the dynamic wrapping and unwrapping of the belt or web that occurs during free oscillations. This action is described by nonlinear boundary conditions, which exist, of course, for translating and nontranslating systems alike. A substantial understanding of such contact for chain/sprocket systems developed early (Mahalingham, 1958), perhaps due to the relatively large impact loads (and noise) induced by discrete polygonal action (Turnbull and Fawcett, 1973; Sueoka, Kondou, and Tanaka, 1989 and Wang, Liu, Hayek, and Chen, 1992). By contrast, relatively little attention has focused on the continuous contact generated in most other axially moving material applications.

Doyle and Hornung noted through experiments that simply supported boundary conditions are inappropriate for V-belts on pulleys (Doyle and Hornung, 1969). The contact of a 
band wrapped about two wheels was analyzed in Hwang and Perkins (1992) by considering globally large static band deflections. Dynamic contact at the belt/pulley interface was analyzed in recent numerical studies (Yue, 1992a,b). The interpretation of these numerical results is facilitated by the present analytical study. Moreover, the differences between the numerical studies by Yue and this investigation are highlighted herein.

The present investigation revisits the problem of dynamic contact and, through application of closed-form analysis, provides a fundamental understanding of the contact-induced nonlinearities. The motivation for this study derives principally from paper forming machines and automotive accessory drive belts wherein changes in web/belt length due to dynamic wrapping and unwrapping may be an appreciable fraction of the steady-state free span length. Finite motions of the element (web/belt) alter the contact location and contact boundary conditions. Such motions are considered herein in the context of two models. The first model approximates the wet paper forming process as a flexible web (string) on an equivalent elastic foundation in contact with a form roll. The second model approximates an accessory drive belt as a tensioned beam contacting a pulley. A common analytical procedure is used to capture the effects of the nonlinear boundary conditions. The present analyses ignore the gyroscopic effects due to web/belt translation speed in order to first focus on the effects of nonlinear contact. The procedures used, however, may be readily extended to include gyroscopic effects.

Section 2 summarizes the initial/boundary value problems governing free response for the two models. The first model, a string on an elastic foundation, is presented in Section 3 and an analytical procedure is developed to evaluate free nonlinear oscillations for this relatively simple, second-order system. Section 4 presents the second model, a tensioned beam, and demonstrates how the procedure generalizes to this fourth-order system. The results and conclusions are presented in Sections 5 and 6, respectively, and serve to highlight the role played by the nonlinear contact for each model.

\section{THE FREE RESPONSE PROBLEM}

The system of interest is defined in Figure 1a. The element shown is considered to be either a belt or a web and is pinned at the (stationary) left boundary and makes contact with a cylindrical surface at the right boundary. The origin of the Cartesian coordinate system is located at the pinned end of the belt and the center of the cylinder is located a distance $L_{0}$ along the positive $X$ direction and $R$ along the negative $Y$ direction. Variables shown in Figure 1 appear in dimensionless form, with the upper-case dimensional variables replaced by their lower-case dimensionless equivalents. The length scale used to normalize distances is $L_{0} / \pi$.

The classical linear equation for free response in nondimensional form is

$$
3 y_{x c x}+y_{t t}-y_{x x}+\delta y=0,
$$

where the time scale is $(\pi \sqrt{T \rho}) / L_{0}$. Here, $T$ is the equilibrium tension, $\rho$ is the belt mass per unit length and the value $\pi$ is introduced such that the wave numbers of the linearized system are integers. The time scale has been selected such that the phase speed 

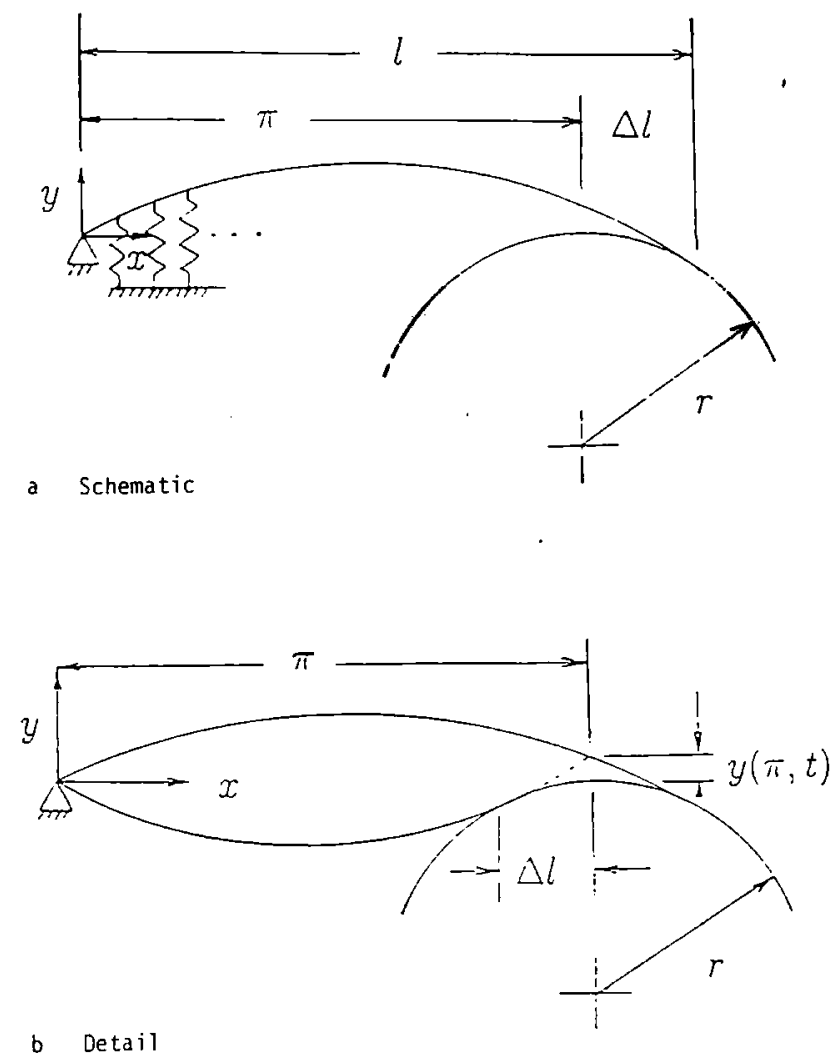

Figure 1. a) A beam or string contacting a circular surface of nondimensional radius $r$. The center of the circle is located a distance $\pi$ along $x$ and $-r$ along $y$. All distances shown are normalized with the length scale $L_{0} / \pi$. b) Detail of the displacement at $x=\pi$ in two deflected positions.

is unity in the limit $\beta \rightarrow 0$ and $\delta \rightarrow 0$. The dimensionless coefficients $\beta=E I \pi^{2} / T L_{0}^{2}$ and $\delta=K L_{0}^{2} / T \pi^{2}$ measure the ratio of the element bending stiffness and foundation stiffness to the tension (membrane stiffness).

The limit $\beta \rightarrow 0$ results in the first model, defining a string on an elastic foundation. The limit $\delta \rightarrow 0$ results in the second model, defining a tensioned beam. The special case $\beta \rightarrow 0$ and $\delta \rightarrow 0$ (a taut string) leads to a seemingly simpler model. This model, however, requires consideration of all modes at lowest order that are drawn in by internal resonances (Kevorkian and Cole, 1981).

Common to both models is the boundary condition at $x=0$ defining an immovable support

$$
y(0, t)=0
$$

In the beam model $(\beta \neq 0)$, the additional boundary condition

$$
y_{\mathrm{rx}}(0, t)=0
$$


The boundary conditions at the opposite end $x=l$ describe the mechanics of a string or beam wrapped around a pulley of nondimensional radius $r=R \pi / L_{0}$. The condition that $y(l, t)$ contact the pulley, applicable to both models, is

$$
y(l, t)=\sqrt{r^{2}-\Delta l^{2}}-r .
$$

The change in the contact region $\Delta l$ is determined by satisfying the condition that the belt remains tangent to the pulley at the contact point. The slope at $x=l$ is the negative reciprocal of the slope of the radial line at the contact point and is therefore

$$
y_{x}(l, t)=\frac{-\Delta l}{\sqrt{r^{2}-\Delta l^{2}}} .
$$

Here, $\Delta l$ is defined as positive to the right (and hence $\Delta l$ as shown in Figure $1 \mathrm{~b}$ for the lower curve is negative). Solving for $\Delta l$ and substituting into (4) yields

$$
y(l, t)=r\left[\sqrt{1-\frac{y_{x}^{2}(l, t)}{1+y_{x}^{2}(l, t)}}-1\right],
$$

where

$$
l=\pi-r \operatorname{sgn}\left(y_{x}(l, t)\right) \sqrt{\frac{y_{x}^{2}(l, t)}{1+y_{x}^{2}(l, t)}}
$$

represents the current nondimensional length of the free belt span. For the tensioned beam model, the belt inherits the curvature of the pulley at $x=l$. Thus

$$
y_{x x}(l, t)=-\frac{1}{r}\left[1+y_{x}^{2}(l, t)\right]^{3 / 2}
$$

is also required in the tensioned beam model.

To complete the free response problem, consider the initial conditions

$$
y(x, 0)=g(x), \quad y_{t}(x, 0)=0,
$$

where $g(x)$ is the initial displacement, which induces an initial energy $\epsilon^{2}$ defined by

$$
\epsilon^{2}=\frac{1}{2} \int_{0}^{\pi}\left[y_{x}^{2}(x, 0)+\beta y_{x x}^{2}(x, 0)+\delta y^{2}(x, 0)\right] d x
$$

The subsequent analysis focuses on weakly nonlinear belt oscillations induced by the above initial energy. To this end, the boundary conditions at $x=l,(6-8)$ are expanded in 
Taylor series about $x=\pi$ assuming small amplitude deflections $y(x, t) \ll 1$. Third-order approximations to (6) and (8) are

$$
\begin{gathered}
y(\pi, t)=r y_{x}^{2}(\pi, t) / 2+r^{2} y_{x x}(\pi, t) y_{x}^{2}(\pi, t)+O\left(y^{4}\right), \\
y_{x x}(\pi, t)=-1 / r+r y_{x x r}(\pi, t) y_{x}(\pi, t)-\frac{6}{r} y_{x}^{2}(\pi, t)+O\left(y^{4}\right),
\end{gathered}
$$

using third-order approximations to (7). Notice that while the displacement at $x=l$ is always negative, the approximate boundary condition, applied at $x=\pi$, is always positive as illustrated in Figure 1b. Equation (11) reveals that the lateral displacement at $x=\pi$ is proportional to the square of the local slope resulting in a quadratic nonlinearity in this boundary condition. A quadratic nonlinearity also appears in (12) arising from the finite beam curvature at the end. In the previous numerical studies (Yue 1992a,b), this boundary condition is linearized. However, the nonlinearity in the curvature boundary condition (12) is of the same order as that in the displacement boundary condition (11). Thus it is inconsistent to linearize the curvature boundary condition while considering the exact or higher-order approximations of the displacement boundary condition.

Note that while the boundary conditions (11) and (12) are nonlinear and quadratic to first nonlinear order, the equation of motion (1) is linear. Specifically, (1) is linearized about the trivial state $y(x, t) \equiv 0$. In the case of the string on an elastic foundation (secondorder system), finite-amplitude motion, resulting in stretching of the string centerline, would contribute cubic nonlinearities to the equation of motion. Thus, for this model, the nonlinear boundary condition (11) is of lower-order and the (cubic) nonlinearities in the equation of motion are presently neglected.

In the case of the tensioned beam model (fourth-order system), consideration of finite amplitude motion and/or finite curvature of the beam centerline would again contribute cubic nonlinearities to the governing equation (1). In this case, however, a non-trivial equilibrium solution also exists (refer to Section 4.1). Further consideration of weakly nonlinear oscillations about this curved equilibrium state results in both quadratic and cubic nonlinearities in the equation of motion. These quadratic nonlinearities scale with the magnitude of the equilibrium curvature, which is bounded by the bending parameter $\beta / r$. As described in Section 4.1, attention will focus on cases where $3 / r \ll 1$ for which the equilibrium solution is essentially trivial. Thus the (quadratic and cubic) nonlinearities in the equation of motion are neglected in favor of the dominant nonlinearities in the boundary conditions (11) and (12).

\section{STRING ON AN ELASTIC FOUNDATION}

For this model the bending rigidity is neglected $(\beta=0)$ and the foundation stiffness is retained $(\delta \neq 0)$. This model is treated in Bhat, Xistis, and Sankar (1982), Perkins (1990), and Tan and Zhang (1994) for linear boundary conditions and is motivated by applications of continuously supported conveyers and paper sheets. 
Present attention will focus on determining the effect of the nonlinear boundary conditions in altering the frequency and mode shape of free oscillation. To this end, the method of strained coordinates, also referred to as the Poincaré-Lindstedt method (Kevorkian and Cole, 1981), is used to evaluate the response problem of Section 2. Substituting $\tau=\omega t$, where $\omega$ is the free oscillation frequency into (1) results in

$$
\omega^{2} y_{\tau \tau}-y_{x x}+\delta y=0
$$

The free oscillation frequency $\omega$ and the displacement $y(x, \tau)$ are expanded in powers of the small amplitude parameter $\epsilon$ :

$$
\omega=\omega_{0}+\epsilon \omega_{1}+\epsilon^{2} \omega_{2}+\cdots
$$

and

$$
y=\epsilon y_{1}+\epsilon^{2} y_{2}+\epsilon^{3} y_{3}+\cdots
$$

Substituting (14) and (15) into (2), (10), (11), and (13), then collecting terms of like order in $\epsilon$ results in the following sequence of linear initial/boundary-value problems.

\subsection{The $O(\epsilon)$ Problem}

The $O(\epsilon)$ problem is

$$
\begin{gathered}
\mathcal{L} y_{1}=0 \\
y_{1}(0, \tau)=y_{1}(\pi, \tau)=0 \\
1=\frac{1}{2} \int_{0}^{\pi}\left[y_{1_{\mathrm{r}}}^{2}(x, 0)+\delta y_{1}^{2}(x, 0)\right] d x .
\end{gathered}
$$

Here,

$$
\mathcal{L} \equiv \omega_{0}^{2} \frac{\partial^{2}}{\partial \tau^{2}}-\frac{\partial^{2}}{\partial x^{2}}+\delta
$$

denotes a linear partial differential operator.

Employing the separable solution

$$
y_{1}=X(x) S(\tau)
$$

results in

$$
\begin{aligned}
& \frac{d^{2} X}{d x^{2}}+\lambda^{2} X=0 \\
& X(0)=X(\pi)=0
\end{aligned}
$$


where $\lambda^{2}$ is the separation constant and

$$
\frac{d^{2} S}{d \tau^{2}}+\frac{\lambda^{2}+\delta}{\omega_{0}^{2}} S=0
$$

The eigenvalue problem (21) and (22) has the elementary characteristic equation

$$
\sin (\lambda \pi)=0
$$

from which $\lambda_{n}=n$ where $n=1,2,3, \ldots$ Thus the general solution for $S(\tau)$ is

$$
S=A_{n} \cos \left(\Omega_{n} \tau\right)+B_{n} \sin \left(\Omega_{n} \tau\right)
$$

where $\Omega_{n}=\sqrt{n^{2}+\delta} / \omega_{0}$. The initial condition is assumed to be an initial displacement $(9 \mathrm{a}, \mathrm{b})$ and therefore $B_{n}=0$. Summing all modal contributions results in

$$
y_{1}=\sum_{n=1}^{\infty} A_{n} \cos \left(\Omega_{n} \tau\right) \sin (n x)
$$

Attention is now restricted to the case where the initial condition to leading order is given by the fundamental mode, $g(x)=A_{1} \sin (x)$. Selecting $\omega_{0}=\sqrt{1+\delta}$, the natural frequency of the fundamental mode, and then substituting (26) into (18) and making use of mode orthogonality leads to

$$
1=\frac{A_{1}^{2}}{2} \int_{0}^{\pi} \cos ^{2}(x)+\delta \sin ^{2}(x) d x
$$

from which $A_{1}=2 / \sqrt{\pi(1+\delta)}$. Thus

$$
y_{1}=\frac{2}{\sqrt{\pi(1+\delta)}} \cos (\tau) \sin (x)
$$

\subsection{The $O\left(\epsilon^{2}\right)$ Problem}

The $O\left(\epsilon^{2}\right)$ problem is

$$
\begin{gathered}
\mathcal{L} y_{2}=-2 \omega_{0} \omega_{1} y_{1 \tau \tau}, \\
y_{2}(0, \tau)=0, \\
y_{2}(\pi, \tau)=\frac{r}{2} y_{l_{r}}^{2}(\pi, \tau)=\alpha[1+\cos (2 \tau)],
\end{gathered}
$$


where $\alpha=\frac{r}{\pi(1+\delta)}$ and

$$
0=\int_{0}^{\pi} y_{l_{x}}(x, 0) y_{2_{x}}(x, 0)+\delta y_{1}(x, 0) y_{2}(x, 0) d x
$$

Note that $y_{\mathrm{I}_{\tau \tau}}$ in (29) is secular since it is not orthogonal to all solutions of the homogeneous equation $\mathcal{L} y_{2}=0$. The uniformly valid expansion (15) exists only upon selecting $\omega_{1}=0$. Thus there is no first-order frequency correction.

The general solution for $y_{2}$ is decomposed into homogeneous and particular components. The particular solution consists of a time-independent term and a term with $2 \tau$ time dependence; refer to the inhomogeneous terms in the boundary condition (30b). The time-independent term is

$$
y_{20}=\alpha \frac{\sinh (\sqrt{\delta} x)}{\sinh (\sqrt{\delta} \pi)}
$$

and the time-dependent term is

$$
y_{22}=\alpha \frac{\sin (\sqrt{4+3 \delta} x)}{\sin (\sqrt{4+3 \delta} \pi)} \cos (2 \tau)
$$

Note that the denominator for $y_{22}$ vanishes for any $\delta=\left(m^{2}-4\right) / 3$ where $m=2,3.4, \ldots$ Under these particular conditions, $\delta=0,5 / 3,4, \ldots$ and $2 \Omega_{1}=\Omega_{m}$. Thus, in these cases, there exists a 2:1 internal resonance between the fundamental mode and mode $m$ (Sanders and Verhulst, 1985) and the expansions (14) and (15) are no longer uniform. These particular cases are not considered here.

The homogeneous solution is described by the eigenfunction expansion:

$$
y_{21}=\sum_{n=1}^{\infty} B_{n} \cos \left(\Omega_{n} \tau\right) \sin (n x)
$$

Substituting the entire solution (32-34) into the initial energy condition (31) and using the orthogonality properties of the eigenfunctions eliminates all terms in the homogeneous solution except that associated with the fundamental mode. The resulting solution is

$$
y_{2}=B_{1} \cos (\tau) \sin (x)+\alpha\left(\frac{\sinh (\sqrt{\delta} x)}{\sinh (\sqrt{\delta} \pi)}+\frac{\sin (\sqrt{4+3 \delta} x)}{\sin (\sqrt{4+3 \delta} \pi)} \cos (2 \tau)\right)
$$

where

$$
B_{1}=\frac{8 r}{3 \pi^{2}(1+\delta)^{2}}
$$




\subsection{The $O\left(\epsilon^{3}\right)$ Problem}

The first correction to the natural frequency due to the nonlinear boundary condition occurs at the $O\left(\epsilon^{3}\right)$ problem:

$$
\begin{gathered}
\mathcal{L} y_{3}=-2 \omega_{0} \omega_{2} y_{1_{r \tau}}, \\
y_{3}(0, \tau)=0 \\
y_{3}(\pi, \tau)=r y_{1_{x}}(\pi, \tau) y_{2_{x}}(\pi, \tau) .
\end{gathered}
$$

Secular terms arise from any terms in the boundary condition at $x=\pi$ that have frequency content equal to that of $y_{1}$. Substituting (28) and (35) into (38b) yields

$$
\begin{aligned}
y_{3}(\pi, \tau) & =-A_{1} r \cos (\tau) \\
& \times\left[-B_{1} \cos (\tau)+\alpha\left(\frac{\sqrt{\delta} \cosh (\sqrt{\delta} \pi)}{\sinh (\sqrt{\delta} \pi)}+\frac{\sqrt{4+3 \delta} \cos (\sqrt{4+3 \delta} \pi)}{\sin (\sqrt{4+3 \delta} \pi)} \cos (2 \tau)\right)\right]
\end{aligned}
$$

Define the constant $C$ as

$$
C=\frac{-r^{2}}{\pi(1+\delta)}\left[\sqrt{\delta} \operatorname{coth}(\sqrt{\delta} \pi)+\frac{\sqrt{4+3 \delta}}{2} \cot (\sqrt{4+3 \delta} \pi)\right] .
$$

Then, $y_{3}(\pi, \tau)$ becomes

$$
y_{3}(\pi, \tau)=C A_{1} \cos (\tau)+N S T
$$

where NST represents all the nonsecular terms. The change of variable

$$
u_{3}=y_{3}-C A_{1} f(x) \cos (\tau)-g(x) N S T
$$

is employed such that $u_{3}$ satisfies homogeneous boundary conditions. This requires $f$ and $g$ to satisfy the boundary conditions

$$
f(0)=g(0)=f(\pi)-1=g(\pi)-1=0,
$$

Substituting (42) into (37) and using (28) results in

$$
\mathcal{L} u_{3}=A_{1}\left[2 \omega_{2} \omega_{0} \sin (x)+C\left(f_{x x}+f\right)\right] \cos (\tau)+N S T .
$$

The secular terms in the brackets are combined by selecting $f$ to be the solution of

$$
f_{x x}+f=D \sin (x)
$$


leading to

$$
f(x)=\frac{-1}{\pi} x \cos (x)
$$

and $D=2 / \pi$.

Thus the solvability condition is

$$
0=2 \omega_{0} \omega_{2}+C D
$$

from which

$$
\omega_{2}=\frac{-C}{\pi \sqrt{1+\delta}} .
$$

This frequency correction will be evaluated further following the present derivation of the frequency correction for the tensioned beam model.

\section{TENSIONED BEAM}

The previous analysis for the string on an elastic foundation model generalizes to the present analysis of a tensioned beam contacting a rigid pulley. This model is motivated by applications employing belts or bands in which the contact point between the pulley and the belt varies with belt deflection. Globally large static deflections are considered by Hwang and Perkins (1992) and weakly nonlinear dynamic deflections are evaluated numerically by Yue (1992a,b). Here, the addition of bending rigidity leads to a nontrivial equilibrium state. Thus analysis of free response about equilibrium begins first with an analysis of the equilibrium state.

\subsection{Equilibrium Analysis}

First consider the static displacement $y_{s}(x)$ of the beam induced by wrapping it around a single pulley. The governing equation (1) reduces to

$$
\beta y_{s_{\text {utu }}}-y_{s_{x x}}=0
$$

augmented by the boundary conditions $(2,3,11$, and 12$)$.

The solution

$$
y_{s}=A \beta \sinh (x / \sqrt{\beta})+C x
$$

satisfies (49) and the linear boundary conditions (2) and (3). The integration constants $A$ and $C$ are selected to satisfy the remaining nonlinear boundary conditions (11) and (12). This results in a pair of coupled quadratic equations for $A$ and $C$ leading to four solutions. The physically realizable solution is selected by imposing an impenetrability condition at the pulley; see examples shown in Figure 2. Observe that for the highly tensioned beam considered in Figure $2(\beta=0.001)$ the curvature of $y_{s}$ is restricted to a boundary-layer 


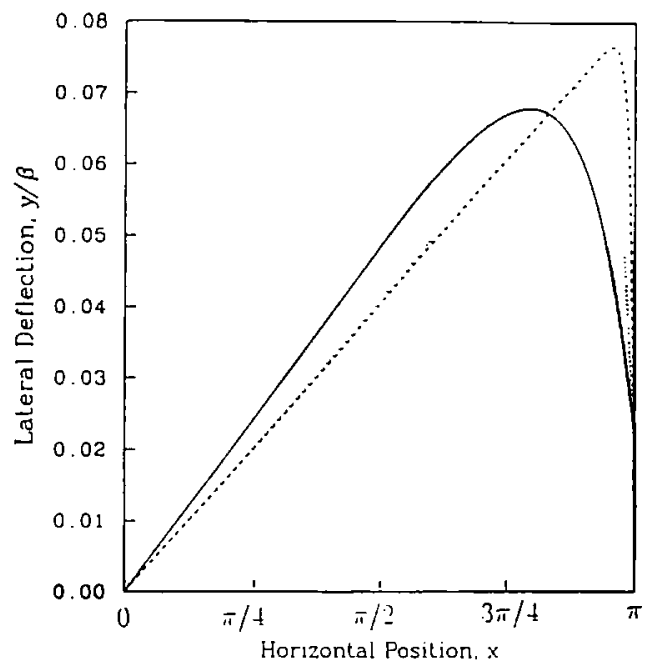

Figure 2. Examples of static equilibrium solutions for $0.1>\beta>0.001$ and pulley radius $r=1$. Key: $\beta=0.1$ $-\beta=0.01 \cdots \cdots(y / \beta) \times 10, \beta=0.001 \cdots(y / \beta) \times 100$.

region near the pulley of length $L_{b l}<0.05 L_{0}$. Even in the case of $\beta=0.1$ the curvature is limited to less than $1 / 3$ of the domain.

The curvature of the equilibrium solution is proportional to the coefficient $A$ in (50) and is bounded from above by the parameter $\beta / r$. As $\beta / r \rightarrow O(1)$, the curvature of the static solution becomes significant throughout the domain. However, the governing equation (1) neglects the geometric nonlinearities, and in particular the quadratic nonlinearities, that affect the weakly nonlinear response about a curved equilibrium state. Because these quadratic nonlinearities scale with the curvature of the static solution, the present model is applicable for the parameter range $\beta / r \ll 1$. In this parameter range, the curvature in the domain is exceptionally small. In reference to Figure 2, observe that in this parameter range the static solution is nearly trivial $(O(\beta))$. Thus this equilibrium is approximated as trivial, which greatly simplifies the mathematical description of subsequent nonlinear oscillations.

\subsection{Free Oscillation}

The beam oscillates freely following a small initial displacement with strain energy $\epsilon^{2}$ defined in (10). Weakly nonlinear oscillations are again examined using the perturbation series (14) and (15). Expanding in powers of $\epsilon$ as before leads to the following sequence of linear initial/boundary-value problems.

\section{3. $O(\epsilon)$ Problem}

The $O(\epsilon)$ problem is

$$
\begin{gathered}
\mathcal{L} y_{1}=0, \\
y_{1}(0, \tau)=y_{1_{x x}}(0, \tau)=y_{1 x x}(\pi, \tau)=y_{1}(\pi, \tau)=0 \\
1=\frac{1}{2} \int_{0}^{\pi}\left[y_{l_{x}}^{2}(x, 0)+\beta y_{l_{u x}}^{2}(x, 0)\right] d x .
\end{gathered}
$$


Here

$$
\mathcal{L} \equiv \beta \frac{\partial^{4}}{\partial x^{4}}+\omega_{0}^{2} \frac{\partial^{2}}{\partial \tau^{2}}-\frac{\partial^{2}}{\partial x^{2}}
$$

denotes a linear partial differential operator.

Separation of variables, with separation constants $\lambda_{n}$, leads to the solution

$$
y_{1}=\sum_{n=1}^{\infty} B_{n} \cos \left(\frac{\lambda_{n}}{\omega_{0}} \tau\right) \sin (n x)
$$

Attention is restricted to the case where the initial condition defines a deflection in the fundamental mode at this order. As in the previous model, higher modes do participate and are subsequently excited by the coupling produced by the nonlinear boundary conditions (11) and (12). Thus $\omega_{0}$ is selected to be the natural frequency of the fundamental mode $\omega_{0} \equiv \lambda_{1}=\sqrt{\beta+1}$. Moreover,

$$
y_{1}=B_{1} \cos (\tau) \sin (x)
$$

where

$$
B_{1}=\frac{2}{\sqrt{\pi(\beta+1)}}
$$

is selected to satisfy the initial energy condition (53).

\section{4. $O\left(\epsilon^{2}\right)$ Problem}

At $O\left(\epsilon^{2}\right)$, the response problem is

$$
\begin{gathered}
\mathcal{L} y_{2}=-2 \omega_{1} \omega_{0} y_{1_{r \tau}}, \\
y_{2}(\pi, \tau)=\frac{r}{2} y_{1_{x}}^{2}(\pi, \tau), \\
y_{2_{u x}}(\pi, \tau)=r y_{1_{x u}}(\pi, \tau) y_{1_{x}}(\pi, \tau)-\frac{6}{r} y_{1_{x}}^{2}(\pi, \tau), \\
y_{2}(0, \tau)=y_{2_{x x}}(0, \tau)=0, \\
0=\int_{0}^{\pi}\left[y_{1_{x}} y_{2_{x}}+\beta y_{1_{x x}} y_{2_{x x}}\right] d x .
\end{gathered}
$$

As before, the term on the right-hand side of (58) is secular and no secular term appears in the boundary conditions (59); thus $\omega_{1}=0$. Using (56), (59a) and (59b) become

$$
\begin{aligned}
& y_{2}(\pi, \tau)=\alpha_{1}[1+\cos (2 \tau)], \\
& y_{2_{\mathrm{u}}}(\pi, \tau)=\alpha_{2}[1+\cos (2 \tau)],
\end{aligned}
$$


where

$$
\begin{gathered}
\alpha_{1}=\frac{r}{\pi(\beta+1)} \\
\alpha_{2}=\frac{-2}{\pi(\beta+1)}\left(r+\frac{6}{r}\right) .
\end{gathered}
$$

The general solution for $y_{2}$ is decomposed into homogeneous and particular components. The particular solution satisfies the non-homogeneous boundary conditions (61) and (62) and can be further decomposed into a time-independent term and the term with $2 \tau$ time dependence. The time-independent term is

$$
y_{20}=\frac{\alpha_{2} \beta \sinh (\sqrt{1 / \beta} x)}{\sinh (\sqrt{1 / \beta} \pi)}+\left(\alpha_{1}-\alpha_{2} \beta\right) \frac{x}{\pi}
$$

and the time-dependent term is

$$
y_{22}=\cos (2 \tau)\left[B_{22} \sin (\hat{a} x)+D_{22} \sinh (\hat{b} x)\right]
$$

where

$$
B_{22}=\frac{\alpha_{1}-\alpha_{2} / \hat{b}^{2}}{\left(1+\hat{a}^{2} / \hat{b}^{2}\right) \sin (\hat{a} \pi)}
$$

and

$$
D_{22}=\frac{\left(\alpha_{1}-\alpha_{2} / \hat{b}^{2}\right) /\left(1+\hat{a}^{2} / \hat{b}^{2}\right)+\alpha_{2}}{\hat{b}^{2} \sinh (\hat{b} \pi)} .
$$

Here the quantities $\hat{a}$ and $\hat{b}$ are

$$
\begin{aligned}
& \hat{a}=\sqrt{\frac{\sqrt{1+8 \beta(\beta+1)}-1}{2 \beta}}, \\
& \hat{b}=\sqrt{\frac{1+\sqrt{1+8 \beta(\beta+1)}}{2 \beta}} .
\end{aligned}
$$

Note that the denominator for $B_{22}$ is singular if $\hat{a}=n$. In general, if $2 \lambda_{1}$ is equal to any of the other eigenvalues $\lambda_{n}(n=2,3, \ldots)$, then $\hat{a}=n$ as was the case in the previous model. However, for the present model, such a 2 : 1 internal resonance exists only for vanishing flexural rigidity $(\beta=0)$. 
The homogeneous solution is described by an eigenfunction expansion of all modes. Substituting the total solution (homogeneous and particular components) into (60) and using eigenfunction orthogonality leads to

$$
y_{2}(x, \tau)=B_{21} \cos (\tau) \sin (x)+y_{20}+y_{22},
$$

where

$$
\begin{aligned}
B_{21} & =\frac{1}{(\beta+1) \pi / 2}\left[\frac{\alpha_{2}(1-\beta)}{(1+1 / \beta)}+\frac{D_{22} \hat{b}^{2}(1-\beta)}{1+\hat{b}^{2}} \sinh (\hat{b} \pi)\right. \\
& \left.-\frac{B_{22} \hat{a}(\beta \hat{a}+1)}{2(1-\hat{a})} \sin ((1-\hat{a}) \pi)-\frac{B_{22} \hat{a}(1-\beta \hat{a})}{2(1+\hat{a})} \sin ((1+\hat{a}) \pi)\right] .
\end{aligned}
$$

\section{5. $O\left(\epsilon^{3}\right)$ Problem}

At third-order, the first correction to the natural frequency due to the nonlinear boundary conditions is determined. At this order, the response problem is

$$
\begin{aligned}
& \mathcal{L} y_{3}=-2 \omega_{2} \omega_{0} y_{1_{\tau}} \\
& y_{3}(\pi, \tau)=r y_{1_{x}}(\pi, \tau) y_{2_{t}}(\pi, \tau)+r^{2} y_{1_{u s}}(\pi, \tau) y_{1_{s}}^{2}(\pi, \tau), \\
& y_{3_{x x}}(\pi, \tau)=r y_{1_{x u t}}(\pi, \tau) y_{2_{\mathrm{s}}}(\pi, \tau)+r y_{2_{u x}}(\pi, \tau) y_{1_{x}}(\pi, \tau)-\frac{3}{r} y_{1_{x}}(\pi, \tau) y_{2_{\mathbf{s}}}(\pi, \tau), \\
& y_{3}(0, \tau)=y_{3_{u}}(0, \tau)=0 .
\end{aligned}
$$

Unlike the $O\left(\epsilon^{2}\right)$ problem, secular terms arise from the boundary conditions and must be eliminated together with the secular term on the right-hand side of (73) as follows. First the nonlinear boundary conditions (74a) and (74b) are examined for terms that have frequency content equal to that of the lowest order solution $y_{1}$. Substituting (56) and (71) into $(74 a)$ yields

$$
y_{3}(\pi, \tau)=-r B_{1} \cos (\tau)\left[y_{20_{x}}(\pi)+y_{22_{x}}(\pi, \tau)-B_{21} \cos (\tau)\right] .
$$

Introducing the constant $C_{1}$

$$
C_{1}=-r\left[y_{20_{\mathbf{r}}}(\pi)+\frac{1}{4}\left(B_{22} \hat{a} \cos (\hat{a} \pi)+D_{22} \hat{b} \cosh (\hat{b} \pi)\right)\right]
$$

permits (75) to be decomposed as

$$
y_{3}(\pi, \tau)=C_{1} B_{1} \cos (\tau)+N S T
$$


where again NST represents all the nonsecular terms. Similarly, (74b) becomes

$$
\begin{aligned}
y_{3_{\mathrm{xx}}}(\pi, \tau) & =(r+3 / r) B_{1} \cos (\tau)\left[y_{20_{x}}(\pi)+y_{22_{x}}(\pi, \tau)-B_{21} \cos (\tau)\right] \\
& -r B_{1} \cos (\tau)\left[y_{20_{x x r}}(\pi)+y_{22_{\mathrm{rux}}}(\pi, \tau)+B_{21} \cos (\tau)\right]
\end{aligned}
$$

Defining $C_{2}$

$$
\begin{aligned}
C_{2} & =(r+3 / r)\left[y_{20_{x}}(\pi)+\frac{1}{4}\left(B_{22} \hat{a} \cos (\hat{a} \pi)+D_{22} \hat{b} \cosh (\hat{b} \pi)\right)\right] \\
& -r\left[y_{20_{\mathrm{ux}}}(\pi)+\frac{1}{4}\left(-B_{22} \hat{a}^{3} \cos (\hat{a} \pi)+D_{22} \hat{b}^{3} \cosh (\hat{b} \pi)\right)\right]
\end{aligned}
$$

results in

$$
y_{3_{\mathrm{xx}}}(\pi, \tau)=C_{2} B_{1} \cos (\tau)+N S T
$$

A variable transformation is now employed, which results in homogeneous boundary conditions for the new unknown $u_{3}$. Select

$$
u_{3}=y_{3}-B_{1}\left(C_{1} f(x)+C_{2} g(x)\right) \cos (\tau)-h(x) N S T
$$

where $f$ and $g$ satisfy the boundary conditions

$$
\begin{aligned}
& f(0)=f_{x x}(0)=f(\pi)-1=f_{x x}(\pi)=0, \\
& g(0)=g_{x x}(0)=g(\pi)=g_{x x}(\pi)-1=0 .
\end{aligned}
$$

Substituting (81) into (73) results in

$$
\begin{aligned}
\mathcal{L} u_{3} & =-2 \omega_{2} \omega_{0} B_{1} \cos (\tau) \sin (x) \\
& -B_{1}\left[C_{1}\left(\beta f_{x x x x}-f_{x x}-\omega_{0}^{2} f\right)+C_{2}\left(\beta g_{x x x x}-g_{x x}-\omega_{0}^{2} g\right)\right] \cos (\tau)+N S T
\end{aligned}
$$

By selecting $f$ and $g$ such that

$$
\beta f_{x x x x}-f_{x x}-\omega_{0}^{2} f=\sin (x)
$$

and

$$
\beta g_{x x x x}-g_{x x}-\omega_{0}^{2} g=\sin (x)
$$


the secular terms can be combined. The functions $f$ and $g$ thus have the form

$$
f=B_{f} \sin (x)+D_{f} \sinh \left(\sqrt{\frac{\beta+1}{\beta} x}\right)+\frac{1}{4 \beta+2} x \cos (x),
$$

where the constants $B_{f}$ and $D_{f}$ are chosen to satisfy the boundary conditions (82c) and (82d) and similarly for the function $g$.

After combining the secular terms, the solvability condition yields

$$
\omega_{2}=\frac{C_{1}+C_{2}}{2 \omega_{0}} .
$$

\section{RESULTS}

\subsection{String on an Elastic Foundation}

Inspection of (40) and (48) reveals that the frequency correction $\omega_{2}$ depends on two parameters: $r$ and $\delta$. The dependence on $r$ is simply that $\omega_{2} \sim r^{2}$ and thus the influence of the nonlinear boundary condition (11) increases with the square of the roll radius $r$. However, the dependence on $\delta$ is not monotonic. This fact is illustrated in Figure 3, which shows how $\omega_{2}$ depends on $\delta$ for the case $r=1$. Note the singularities that exist at $\delta=0,5 / 3,4, \ldots$ These singular points correspond to particular values of $\delta$ for which there is an internal resonance with the fundamental mode. For instance, $\delta=0$ corresponds to $2 \Omega_{1}=\Omega_{2}, \delta=5 / 3$ corresponds to $2 \Omega_{1}=\Omega_{3}$, and so on. The expansions (14) and (15) are no longer uniform in a neighborhood about these particular values of $\delta$. Between these values of $\delta, \omega_{2}$ decreases monotonically with increasing $\delta$.

Recall that the initial conditions considered herein excite exclusively the fundamental mode at first-order. Higher-order linear modes, however, are drawn into the response through the nonlinear boundary condition (11). The particular higher-order mode that participates at order $\epsilon^{2}$ depends strongly on the value of $\delta$. Figure 4 compares the nonlinear mode shape $y=\epsilon y_{1}+\epsilon^{2} y_{2}$ and the linear mode shape $\epsilon y_{1}$ for an example case for which $\delta=0.01, \epsilon=0.01$, and $r=1$. Observe that $y(\pi) \neq 0$ for the nonlinear mode as required by the nonlinear boundary condition (11). For $\delta \rightarrow 0, y_{2}$ converges to the second mode. This is already apparent from Figure $4(\delta=0.01)$ because $y$ crosses $y_{1}$ only once near $x=\pi / 2$. Figures 5 and 6 illustrate how other linear modes are drawn in depending on the value of $\delta$. For $\delta$ slightly less than $5 / 3$ as in Figure $5, y$ crosses $y_{1}$ twice near $x=\pi / 3$ and $x=2 \pi / 3$ as $y_{2}$ resembles the third linear mode. For $\delta$ slightly greater than $5 / 3$ as in Figure 6 there is again evidence of the third linear mode, though the phase of $y_{2}$ is now opposite that of Figure 5. As $\delta$ approaches each higher-order internal resonance, $y_{2}$ approaches that of each higher-order linear mode in sequence.

In the motivating problem of the paper web contacting a form roll, web oscillations may alter the forming length due to the nonlinear boundary condition considered herein. Such dynamic changes in forming length have serious implications for product variability 


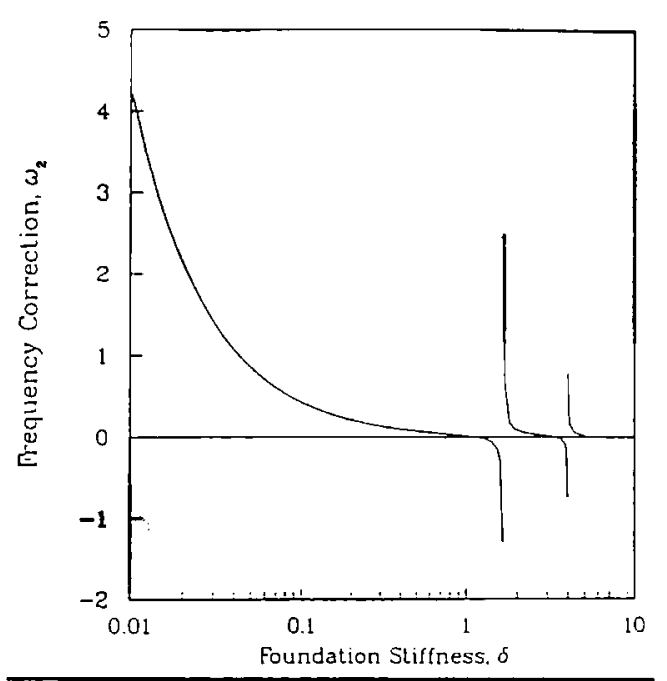

Figure 3. Frequency correction $\omega_{2}$ versus $\delta$ for string on an elastic foundation. In this example, $r=1$.

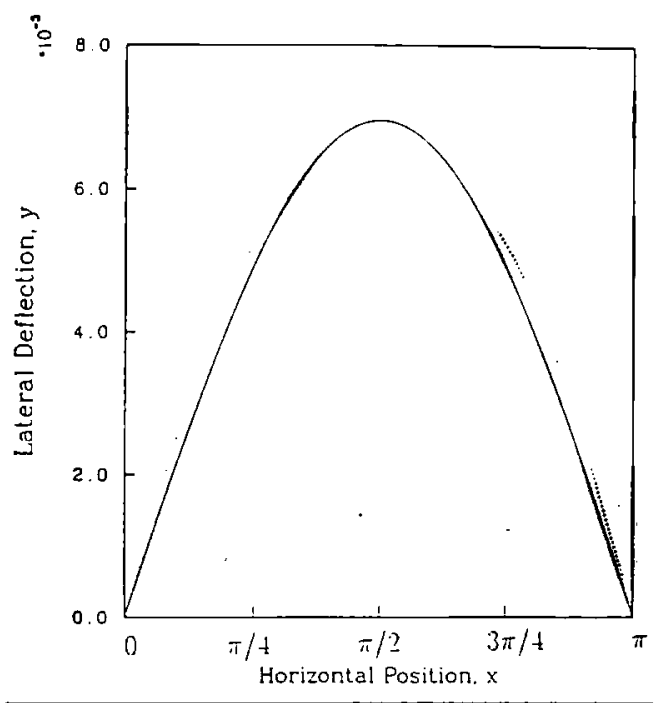

Figure 5. Nonlinear (.....) and linear ( - ) mode shapes for string on an elastic foundation. In this example, $\delta=1.65, r=1$ and $\epsilon=0.01$.

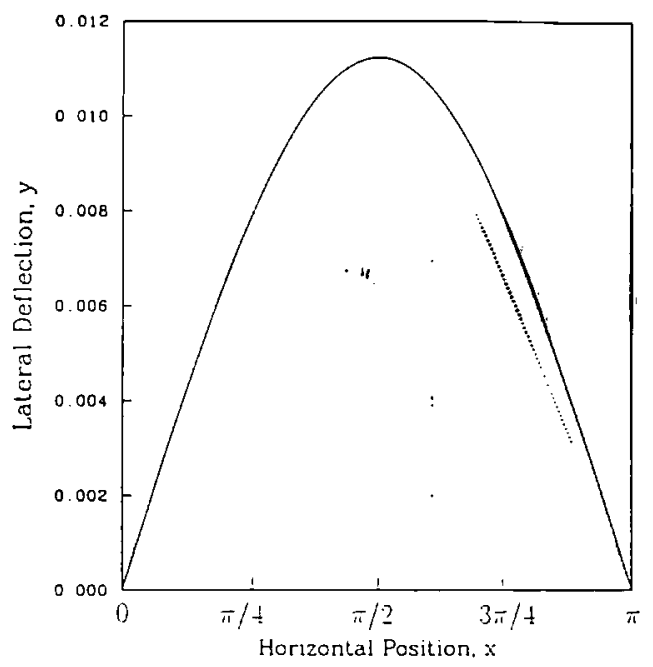

Figure 4. Nonlinear mode shape $y=\epsilon y_{1}+\epsilon^{2} y_{2}$ $(\cdots$.$) compared to the linear mode shape \epsilon y_{1}(\longrightarrow)$ for string on an elastic foundation. In this example $\delta=0.01, r=1$, and $\epsilon=0.01$.

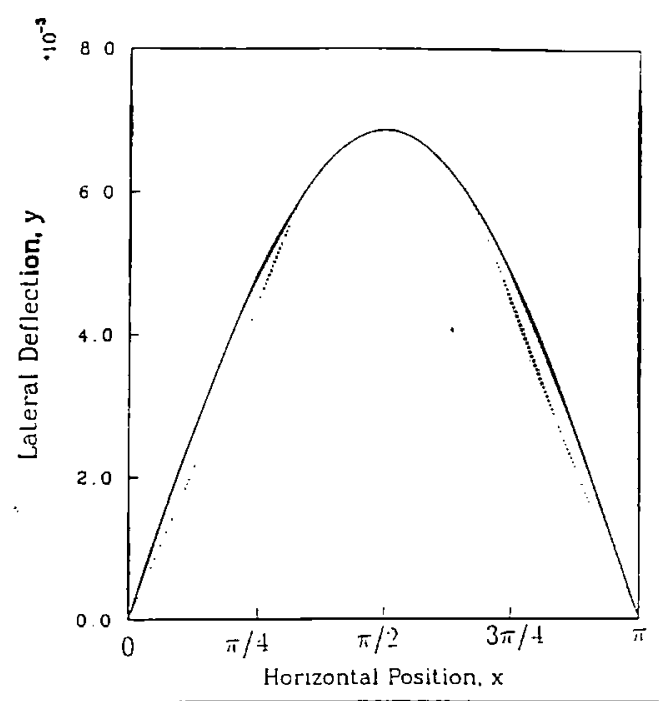

Figure 6. Nonlinear $(. .$.$) ) and linear (-)$ mode shapes for string on an elastic foundation. In this example, $\delta=1.68, r=1$ and $\epsilon=0.01$.

(e.g., thickness) and quality. An estimate of this dynamic length change is

$$
\Delta l=\frac{\Delta L \pi}{L_{0}}=r \sqrt{\frac{y_{x}^{2}(\pi, t)}{1+y_{x}^{2}(\pi, t)}}
$$

(refer to [5]). Figure 7 illustrates how this estimated $\Delta l$ depends on $\delta$ for the example case $r=1$ and $\epsilon=0.01$. For the range $0.02<\delta<0.4$, the change in length is 


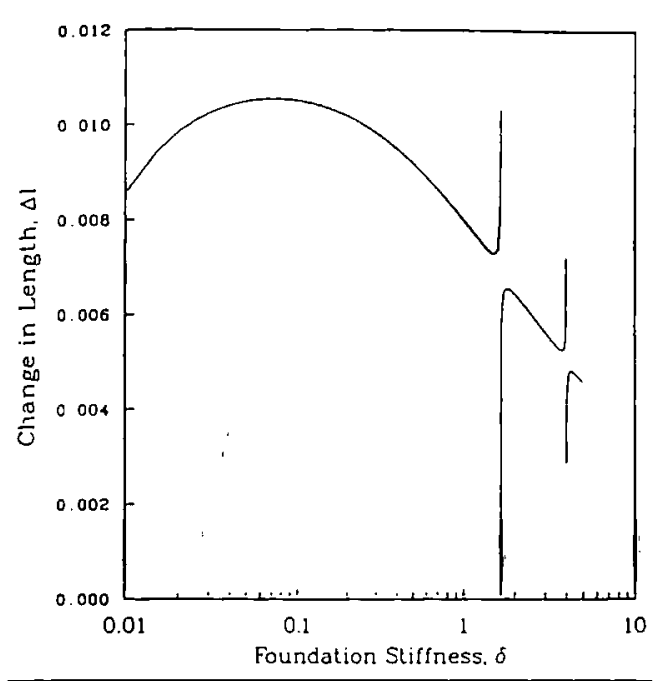

Figure 7. Change in length $\Delta /$ versus $\delta$ for string on an elastic foundation. In this example, $r=1$ and $\epsilon=0.01$.

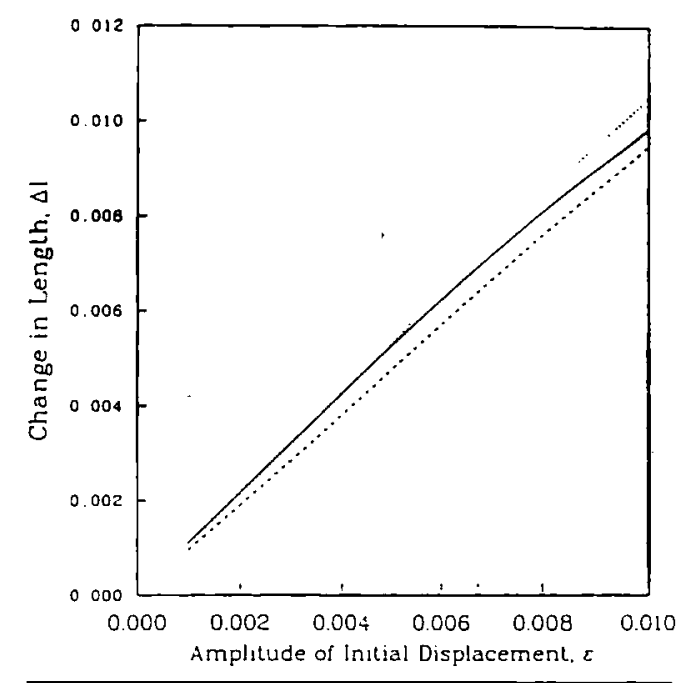

Figure 8. Change in length $\Delta /$ versus $\epsilon$ for string on an elastic foundation. In this example $0.02<\delta<0.4$ and $r=1$. Key: $\delta=0.02-, \delta=0.1 \ldots \ldots, \delta=0.4$ -....

approximated within $10 \%$ with the simple linear relation $\Delta l \approx \epsilon r$. This approximation is readily observable in Figure 8, illustrating the change in length versus $\epsilon$ for several values of $\delta$.

\subsection{Tensioned Beam}

Inspection of (87) shows that $\omega_{2}$ is a function of $r$ and $\beta$ through the quantities $C_{1}$ (76) and $C_{2}$ (79). Further examination of these quantities reveals that $C_{1} \sim r^{2}$ whereas $C_{2} \sim r^{2}+1 / r^{2}$. The constant $C_{1}$ results from the application of the displacement boundary condition (11) and is thus analogous to the constant $C$ for the model of a string on an elastic foundation $(38 \mathrm{~b}-40)$. The constant $C_{2}$, which embodies information from the (higherorder) curvature boundary condition (12), introduces a non-monotonic dependence on $r$ as illustrated in Figure 9 for the case $\beta=0.01$.

The dependence of the frequency correction $\omega_{2}$ on $\beta$ is also non-monotonic as illustrated in Figure 10 for the case $r=1$. In this example, the frequency correction reaches a maximum near $\beta=0.002$ and then decreases with further increase in flexural rigidity $\beta$.

The nonlinear mode shape $y=\epsilon y_{1}+\epsilon^{2} y_{2}$ is plotted for three values of $\beta$ in Figure 11 for the case $\epsilon=0.01$ and $r=1$. In contrast to the elastic foundation model, internal resonances do not exist for the tensioned beam model except, of course, for the degenerate case $\beta=0$. Thus, in this model, only the second linear mode participates at order $\epsilon^{2}$. Higher modes enter only at corresponding higher-orders of $\epsilon$. Note that the magnitude of the second linear mode increases with decreasing flexural rigidity $\beta$ and begins to resemble that for the string/foundation model of Figure 4.

Similarly, the change in length $\Delta l$ increases dramatically with decreasing flexural rigidity $\beta$. This trend is illustrated in Figure 12 where $\Delta l$ is plotted versus $\beta$ for the case $r=1, \epsilon=0.01$. Here again, a simple linear relation $\Delta l \approx 1.15 \epsilon r$ approximates 


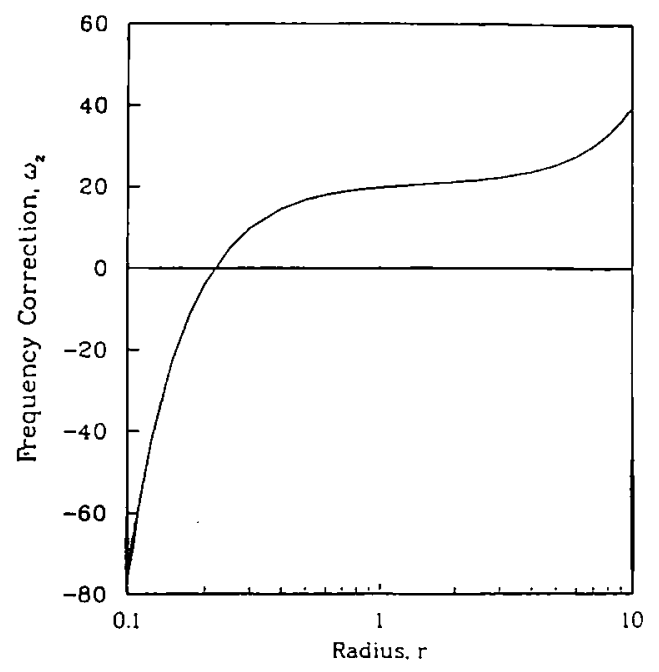

Figure 9 . Frequency correction $\omega_{2}$ versus radius $r$ for tensioned beam. In this example, $\beta=0.01$.

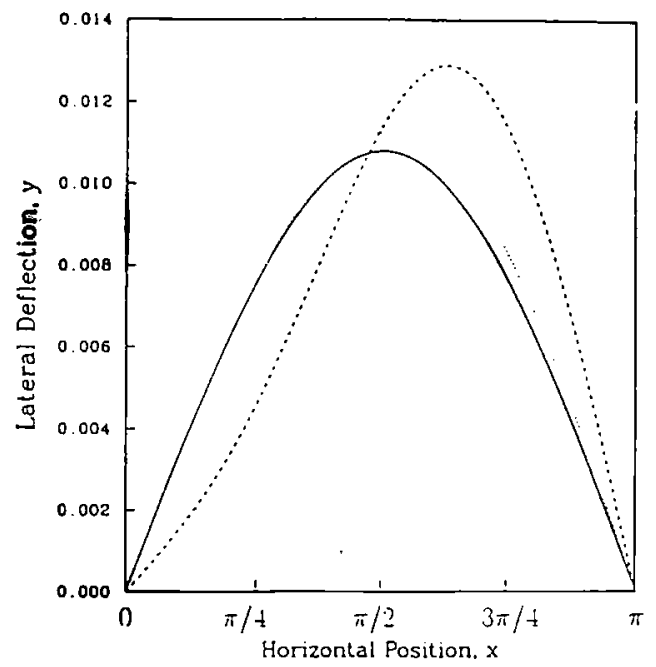

Figure 11. Nonlinear mode shape $y$ versus position $x$ for tensioned beam. In this example $0.001<\beta<0.1$, $r=1$ and $\epsilon=0.01$. Key: $\beta=0.1 \longrightarrow, \beta=0.01$ $\cdots \cdots, \beta=0.001 \cdots$.

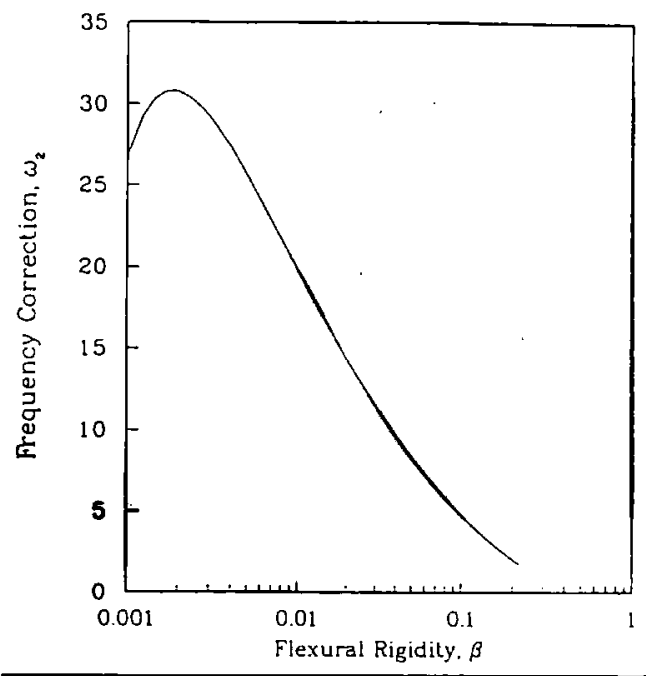

Figure 10. Frequency correction $\omega_{2}$ versus 3 for tensioned beam. In this example, $r=1$.

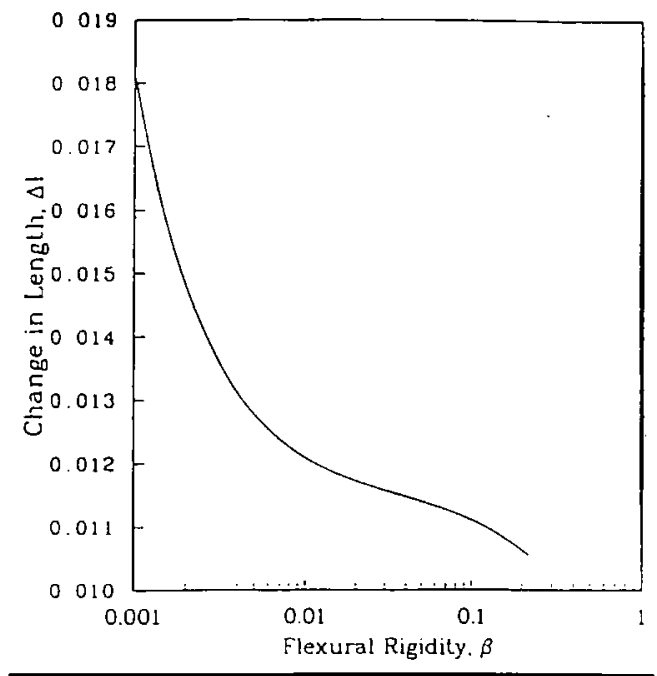

$\overline{\text { Figure 12. Change in length } \Delta / \text { versus } \beta \text { for tensioned }}$ beam. In this example, $r=1$ and $\epsilon=0.01$.

the change in length for the range $0.01<\beta<0.1$ within an error of $10 \%$. This result agrees with the numerical studies by Yue (1992a,b), who also found that the so-called vibrating length is proportional to the pulley radius $r$. However, the dependence of the natural frequency and vibrating length on the strain energy and stiffness parameters are not presented in the studies by Yue (1992a,b). Note also that in automotive accessory drive applications, dynamic length changes create dynamic fluctuations in belt/pulley contact and thus dynamic fluctuations in belt/pulley traction. Moreover, dynamic belt/pulley traction may promote belt slip, chirp, and squeal (Hwang, Perkins, and Ulsoy, 1994). 


\section{CONCLUSIONS}

Two models are presented for evaluating the dynamic wrapping and unwrapping of an element (web or belt) on a smooth surface (roll or pulley). The first model is motivated by the wet paper forming process and consists of an elastically supported string (web or wire) contacting a form roll. The second model is motivated by automotive belt applications and consists of a tensioned beam (belt) contacting a pulley. Modeling of weakly nonlinear oscillations reveals that dynamic contact introduces quadratically nonlinear boundary conditions to first nonlinear order. Subsequent analysis of free nonlinear oscillations reveals that the first correction to the natural frequency $\omega_{2}$ depends solely on two parameters. For the string on an elastic foundation, these parameters are the (nondimensional) pulley radius $r$ and the foundation stiffness $\delta$. For the tensioned beam, these parameters are the (nondimensional) pulley radius $r$ and the bending rigidity $\beta$.

For both models, energy, which originates in the fundamental linear mode, ultimately excites higher-order linear modes. For the string on an elastic foundation, the particular higher-order linear mode that participates at the first nonlinear order $\left(\epsilon^{2}\right)$ depends on the foundation stiffness $\delta$. Internal resonances separate the regions of $\delta$ associated with each higher-order linear mode. By contrast, internal resonances do not exist for the tensioned beam model and the first linear mode ultimately excites only the second linear mode to first nonlinear order $\left(\epsilon^{2}\right)$. For both models the stiffness parameters $\delta$ and $\beta$ strongly influence the amplitude of the higher-order linear modes.

The change in free span length $\Delta l$ due to dynamic wrapping and unwrapping is of interest in both paper forming and automotive belt applications. The models analyzed herein reveal that $\Delta l$ is proportional to the pulley radius $r$ and the amplitude of oscillation $\epsilon$ as determined by the initial conditions. The dependence on the stiffness parameters $\delta$ and $\beta$ is not, however, monotonic.

Acknowledgment. The authors wish to acknowledge the Technical Association of the Pulp and Paper Industry (TAPPI Foundation) and the Ford Motor Company for partial support of this research endeavor.

\section{REFERENCES}

Bhat, R. B., Xistis, F. D., and Sankar, T. S., 1982, "Dynamic behavior of an axially moving belt supported on elastic foundation," ASME Joumal of Mechanical Design 104, 143-147.

Doyle, E. and Hornung, K. G., 1969, "Lateral vibration of v-belts," ASME Paper \#69-VIBR-29 in the Vibration Conference, Philadelphia, PA, March 30-April 2.

Hwang. S. J. and Perkins, N. C., 1992, "High speed stability of coupled band/wheel systems: theory and experiment," Journal of Sound and Vibration 169, 459-483.

Hwang. S. J., Perkins, N. C., and Ulsoy, A. G., 1994, "Rotational response and slip prediction of serpentine belt drive systems." ASME Journal of Vibration and Acoustics 116, 71-78.

Kevorkian, J, and Cole, J. D., 1981, Perturbation Methods in Applied Mathematicr, Springer-Verlag, New York, $448-480$.

Mahalingham, S., 1958, "Polygonal action in chain drives," Journal of Franklin Institure 265(1), 23-28.

Mote, C. D. Jr., 1972, "Dynamic stability of axially moving materials," Shock and Vibration Digest 4, $2-11$.

Perkins, N. C., 1990, "Linear dynamics of a translating string on an elastic foundation," ASME Journal of Vibration and Acoustics 112, 2-7.

Sanders, J. A. and Verhulst F., 1985, Averaging Methods in Nonlinear Dynamical Systems, Springer-Verlag, New York, $149-154$

Sueoka, A., Kondou, T., and Tanaka, T., 1989, "Nonlinear harmonic and parametric resonances of a roller chain stretched horizontally," JSME International Journal 32(3), 385-393. 
Tan, C. A. and Zhang, J., 1994, "Dynamic characteristics of a constrained string translating across an elastic foundation," ASME Journal of Vibration and Acoustics 116, 318-325.

Tumbull, S. R. and Fawcen, J. N., 1973. "Dynamic behavior of roller chain drives," Institute Mechanical Engineers, 29-35. Wang, K. W., Liu, S. P., Hayek, S. I., and Chen, F.H.K., 1992, "On the impact intensity of vibrating axially moving roller chains," ASME Joumal of Vibrarion and Acoustics 114, 397-403.

Wickert, J. A. and Mote, C. D. Jr., 1988, "Current research on the vibration and stability of axially moving materials," Shock and Vibration Digest 20, 3-13.

Yue, M. G., 1992a, "Belt vibration considering moving contact and parametric excitation," ASME 6th International Power Transmission and Gearing Conference $43(1), 311-318$.

Yue, M. G., 1992b, "Moving contact and coupling in belt strand vibration," 14 th ASME Conference of Mechanical Vibration and Noise 59, 139-144. 